\title{
Anatomía Radiológica y realidad aumentada: metodología basada en la ludificación para su aprendizaje
}

\section{Dugnol Menéndez $\mathbf{J}^{\mathrm{a}}$, Jiménez Arberas $\mathbf{E}^{\mathrm{b}}$, Ruiz Fernández $\mathbf{M L}^{\mathrm{c}}$, Fernández Valera $\mathrm{D}^{\mathrm{d}}$ y Merayo- Lloves JM $\mathbf{~}^{\mathbf{e}}$}

${ }^{a}$ Licenciada en Biología por la Universidad de Oviedo. Profesora de prácticas de Anatomía Humana Facultad Padre Ossó y profesora interina de Formación Profesional. Estudiante de Doctorado del Programa Ciencias de la Salud, Universidad de Oviedo; judugnol@gmail.com. ${ }^{b}$ Doctora en Psicología por la Universidad de Salamanca, Terapeuta Ocupacional por la Universidad de Salamanca. Profesora y coordinadora del Grado de Terapia Ocupacional, Facultad Padre Ossó. Universidad de Oviedo; estibaliz@facultadpadreosso.es. ${ }^{\mathrm{d}}$ Doctora en Medicina y Cirugía por la Universidad de Oviedo. Médico Especialista en Medicina de la Educación Física y el Deporte. Profesora de Anatomía Humana y Geriatría y Patologías Osteoarticulares. Grado Terapia Ocupacional. Facultad Padre Ossó. Universidad de Oviedo; luisa@ffacultadpadreosso.es. ${ }^{\mathrm{d} A l u m n o ~ G r a d o ~ F a c u l t a d ~ d e ~ M e d i c i n a, ~ U n i v e r s i d a d ~ d e ~ O v i e d o ; ~}$ UO238069@uniovi.es. ${ }^{\mathrm{C} C a t e d r a ́ t i c o ~ e n ~ O f t a l m o l o g i ́ a ~ p o r ~ l a ~ U n i v e r s i d a d ~ d e ~ O v i e d o . ~ M e ́ d i c o ~ e s p e c i a l i s t a ~ e n ~}$ Oftalmología y profesor titular de Oftalmología en el Grado de Medicina, Universidad de Oviedo; merayojesus@uniovi.es.

\begin{abstract}
In recent years, gamification in the classroom has gone from being used primarily in the school environments to also being used at universities. The present work shows and compares a gamification experience carried out in students belonging to both higher education and nonuniversity higher education. It is a questionnaire-type game based on real clinical cases of Radiology and Radiological Anatomy, in which the student advances as the answer shown through augmented reality is correct. After playing, the theoretical-practical knowledge acquired will be analyzed and statistically significant improvements will be observed. Finally, satisfaction surveys indicate that both student profiles prefer to use gamification tools in their learning and the development of their skills.
\end{abstract}

Keywords: Gamification, radiological anatomy, Occupational Therapy, Certificate of Higher Education

\section{Resumen}

En los últimos años, la ludificación en el aula ha pasado de ser usada exclusivamente en el ámbito escolar hasta llegar a la universidad. El presente trabajo muestra y compara una experiencia de ludificación llevada a cabo en alumnado perteneciente tanto a Educación Superior universitaria como no universitaria. Se trata de un juego tipo quizz basado en casos clínicos reales de Radiología y Anatomía Radiológica, en los que el estudiante va avanzando a medida que acierta la respuesta mostrada a través de realidad aumentada. Tras el juego, se analizaron los conocimientos teórico-prácticos adquiridos y se observaron mejoras estadísticamente significativas. Por último, las encuestas de satisfacción indican que ambos perfiles de alumnado prefieren usar herramientas de ludificación en su aprendizaje y en el desarrollo de sus competencias.

Palabras clave: Ludificación, Anatomía Radiológica, Terapia Ocupacional, Ciclo Formativo de Grado Superior 


\section{Introducción}

Actualmente, el concepto de enseñanza en Educación Superior (tanto universitaria como no universitaria) se está centrando en la búsqueda de un aprendizaje más activo y centrado en el alumnado. Se busca no sólo alcanzar los conocimientos necesarios sino también fomentar el desarrollo de las personas, sus habilidades sociales y curriculares, que son objetivos difíciles de alcanzar con los métodos tradicionales (Andreu-Andrés y García-Casas, 2014).

Una de las herramientas de enseñanza que más ha interesado a la comunidad docente, en todos los niveles educativos, es la ludificación (Rutledge et al., 2018). Aunque existen diferentes definiciones, de forma general, el término se refiere al uso de elementos y mecánicas de juego en situaciones no recreativas, como en un aula; se imita la estética y las estrategias del juego para promover el aprendizaje y la resolución de problemas (Kapp, 2012). Este tipo de técnicas se están introduciendo en el campo educativo dando lugar a lo que se conoce como ludificación educativa (Domínguez et al., 2013) y tiene como objetivo influir en el comportamiento del alumnado para motivarle, puesto que la motivación es un factor determinante en los procesos cognitivos (Contreras-Espinoza, 2016).

Por tanto, los juegos se han transformado en una herramienta eficaz para aprender y abordar tareas difíciles o complicadas promoviendo, entre otras cosas, el trabajo en equipo (Hoffman y Nadelson, 2009; Monaghan y Nicholson, 2017). Las dinámicas del juego se han implementado desde Educación Infantil hasta llegar a los programas de educación superior universitarios. De esta forma, los juegos de estrategia, juegos de rol y los juegos didácticos con contenidos de aprendizaje de la materia, cambian la dinámica de las clases meramente expositivas e incluso de las prácticas de aula o seminarios (Errington, 2001). Si además el juego permite la cooperación y la colaboración, el resultado implica no solo obtener más conocimiento, sino mejorar otras competencias como son el trabajo en equipo, la comunicación y la resolución de problemas (Fukuchi et al., 2000), que son esenciales para los estudiantes y profesionales de Ciencias de la Salud. La introducción de la ludificación en el aula radica en la importancia que tiene la propia participación del alumno en su aprendizaje y en la retroalimentación directa e inmediata que recibe cuando juega.

El uso de metodologías de aprendizaje más innovadoras y activas cada vez está más al alcance del profesorado por la existencia de diferentes tipos de aplicaciones disponibles; además, los estudiantes de hoy en día están acostumbrados a vivir en un mundo altamente tecnológico (Baid y Lambert, 2010). Por ello, se pensó en el diseño de un juego de tipo quizz en formato analógico-digital en el que el alumnado pudiera ir avanzando en los casos clínicos propuestos (sobre Anatomía Radiológica y Radiología en general) al ir acertando las preguntas y, además, recibiera un feedback a tiempo real de las posibles opciones de respuesta y aprendiera de sus errores.

\section{Objetivos}

El objetivo principal es implementar la ludificación en el aula para el aprendizaje de un determinado contenido referente a Anatomía Radiológica tanto en Educación Superior universitaria como no universitaria. Se pretende incrementar de esta forma la motivación del alumnado y del profesorado, así como analizar la valoración de dicha experiencia por parte de los estudiantes. Para ello, nos planteamos los siguientes objetivos específicos:

- Utilizar herramientas y aplicaciones tecnológicas al servicio de la propuesta metodológica. 
- Trabajar no solo los conocimientos sino también las competencias transversales fundamentales en el área de las Ciencias de la Salud.

- Identificar si existen diferencias significativas entre el aprendizaje individual y/o grupal tras la experiencia de ludificación.

- Conocer la opinión de los estudiantes sobre la ludificación, aplicación de los conocimientos y competencias, así como de la herramienta usada para el aprendizaje e identificar si existen diferencias estadísticamente significativas entre ambos perfiles de estudiantes.

\section{Desarrollo de la innovación}

\subsection{Plan de trabajo desarrollado}

Primeramente, se reunieron los docentes que participarían en la actividad, para la distribución de las tareas y funciones de cada uno. Se realizó una revisión bibliográfica para poder diseñar un juego quiz educativo que combinase un formato analógico y otro digital, mediante el uso de una App de realidad aumentada. El juego se desarrolló para poder adaptarse a cualquier tipo de materia o contenido. Fue diseñado para evaluar las habilidades técnicas y el conocimiento anatómico-radiológico y clínico; pero además, se quería observar el razonamiento y el uso de las competencias básicas del alumnado durante el transcurso del juego. El desarrollo del plan de trabajo queda reflejado de manera sencilla en la tabla 1.

Tabla 1. Planificación del trabajo.

\section{ANÁLISIS}

- Recogida de información.

- Análisis de bibliografía disponible y contexto de aplicación.

- Búsqueda de Apps gratuitas.

\section{DISEÑO}

- Selección de contenidos, objetivos didácticos a alcanzar y competencias.

- Creación de los casos clínicos de radiología.

- Estructuración del test secuencial y establecimiento de las reglas del juego.

- Boceto de dibujos de los personajes del juego.

- Creación del material: tarjetas de respuesta, diapositivas/imágenes de cada posible opción del juego, subida a la aplicación de realidad aumentada HP Reveal de las respuestas.

- Creación de los cuestionarios pre-juego y post-juego.

DESARROLLO DE LA VERSIÓN PILOTO CON ESTUDIANTES DE $1^{\circ}$ DE TERAPIA OCUPACIONAL

- Explicación de las reglas del juego y preparación del material..

- Creación de los grupos de juego. 
- Reparto y recogida de los cuestionarios pre-juego grupales.

- Actividad de ludificación: para ello los alumnos se descargan la App gratuita.

- Reparto y recogida de los cuestionarios post-juego grupales.

- Resolución de dudas.

- Envío de encuestas de satisfacción

- Análisis de los resultados.

\section{REDISEÑO DEL JUEGO: APLICACIÓN EN ESTUDIANTES DE FORMACIÓN PROFESIONAL}

- Tras probar el piloto, se tuvo que realizar una serie de modificaciones:

○ Elección de un grupo más reducido de participantes.

○ Los cuestionarios pre-juego se harían de manera individual.

- Se les repartieron de manera simultánea los cuatro casos para agilizar el desarrollo del juego.

- Los cuestionarios post-juego primeramente se resolvieron de manera individual y, posteriormente, de manera grupal.

- Resolución de dudas.

- Envío de encuestas de satisfacción de manera inmediata, para conseguir mayor cantidad de respuestas.

○ $\quad$ Análisis de los resultados.

\subsection{Descripción de la metodología}

El piloto del juego Lucusines $@$ se planteó por primera vez en el grado de Terapia Ocupacional (TO) de la Facultad Padre Ossó, con 37 estudiantes matriculados en la asignatura Anatomía Humana (74\% de los matriculados) en un seminario sobre Anatomía Radiológica, debido a que dichos estudiantes deben tener unos conocimientos mínimos de interpretación de imágenes radiológicas para otras asignaturas del grado. Se planteó como actividad voluntaria.

Una vez testado por primera vez, se observaron las posibles mejoras tanto del juego como del diseño. Tras ser revisado, se jugó con otro perfil de alumnado de Educación Superior. En este caso, fueron estudiantes de Educación Superior no Universitaria; en concreto, se escogieron estudiantes de Formación Profesional (FP) de Grado Superior del Ciclo Imagen para el Diagnóstico y Medicina Nuclear del centro de Svida Formación. Participaron voluntariamente 16 estudiantes que estaban cursando el primer curso de dicho ciclo de FP (66,67\% de los matriculados). La elección de este grupo de estudiantes fue debido a que el juego creado inicialmente versaba sobre Anatomía Radiológica y conceptos básicos de radiología, por lo que fue implementado en el módulo de Anatomía por la Imagen del ciclo formativo.

Antes de jugar, en ambas ocasiones, se explicaron las reglas y se les indicó qué aplicación de realidad aumentada debían descargarse para jugar (HP Reveal, de la App Store) y cómo se usaba.

La aplicación consiste en la creación de "auras", en las que se toma una foto de un objeto y se le asocia una imagen oculta, que podrá visualizarse si se escanea con el programa dicho objeto. Para ello, antes del juego, se fueron creando tarjetas de respuesta y creando las auras con las capturas de pantalla del juego Lucusiones $^{\mathcal{O}}$ para preparar todo el material. 
El juego comienza una vez se tenga descargada la aplicación y se hayan repartido los diferentes casos clínicos, con su descripción y su primera pregunta. Cuando el alumnado decide cuál es la respuesta, se le ofrece la tarjeta de su opción de respuesta para que la escanee y pueda seguir avanzando en el juego (Figs. $1-3)$.
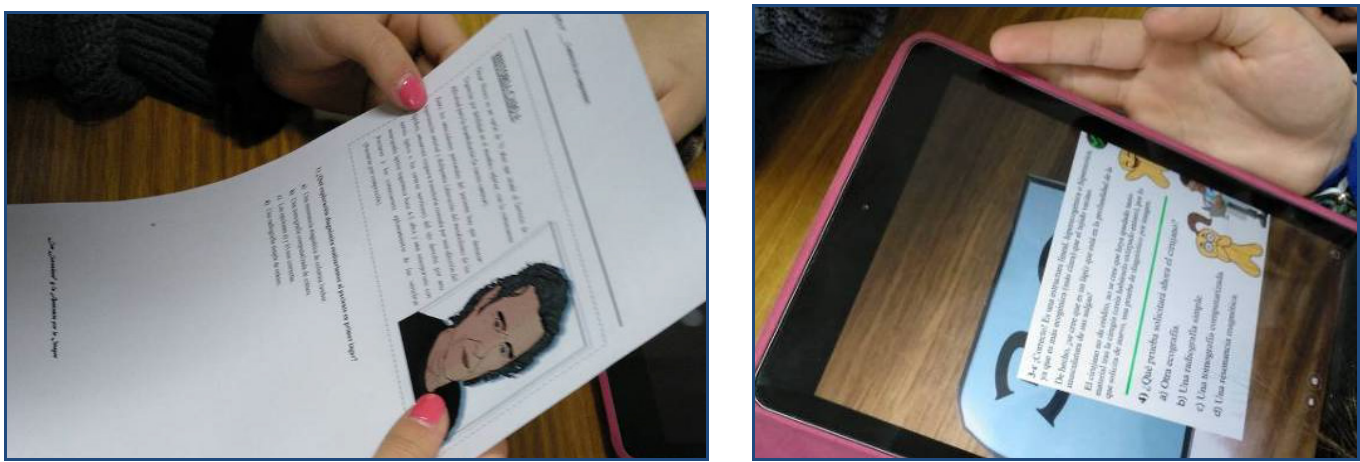

Fig. 1 Las imágenes muestran a estudiantes de TO leyendo el último caso clínico (izquierda) y usando la app de realidad aumentada para comprobar si su respuesta era correcta y avanzar para poder resolver el caso (derecha).
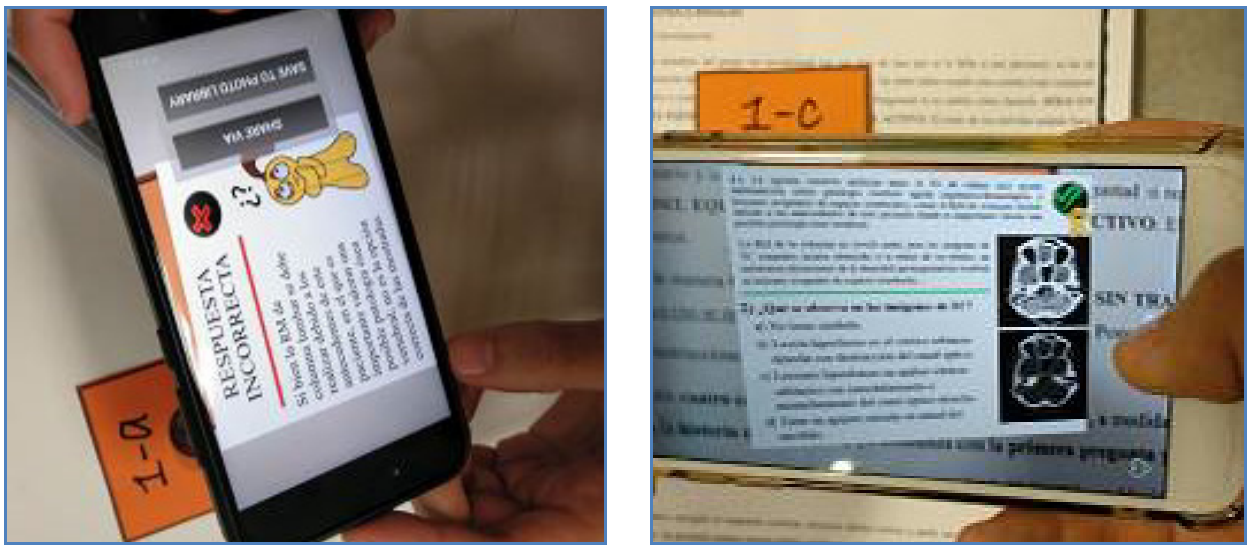

Fig. 2 Imágenes de realidad aumentada alojadas en la App. A la izquierda, se observa que, tras escanear la tarjeta elegida, les sale la información de que su opción es incorrecta con su justificación. A la derecha, se escaneó la opción correcta, por lo que el programa les muestra la siguiente pantalla que plante la siguiente pregunta.
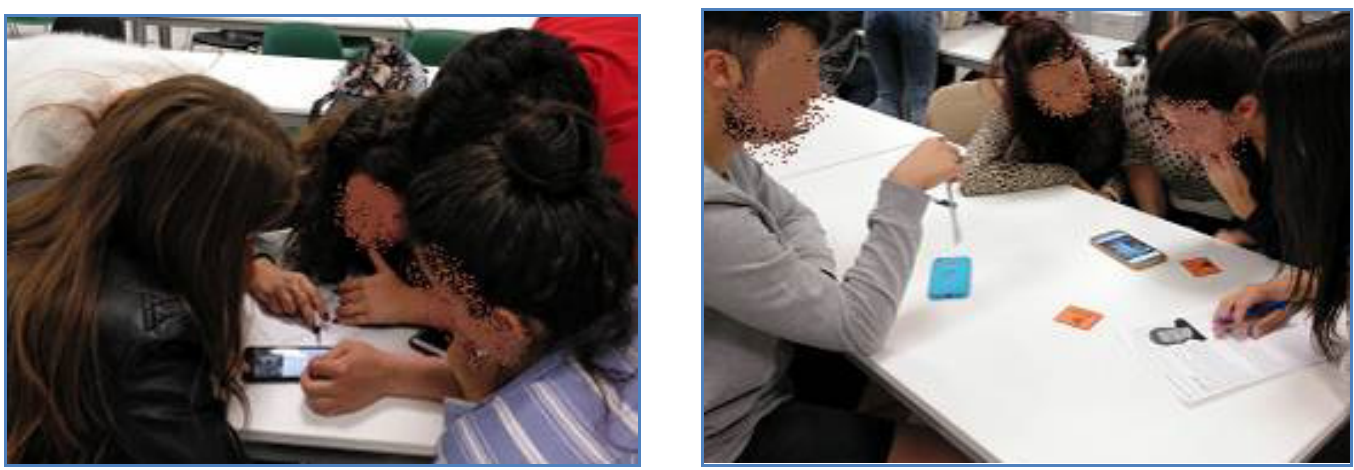

Fig. 3 Alumnos de Formación Profesional resolviendo los casos con la App.

En cada actividad de ludificación se desarrolló un cuestionario para comprobar el nivel de acierto, antes y después del juego (esta última, de manera individual y, posteriormente, grupal en el caso de los estudiantes de FP; mientras que los estudiantes de TO respondieron grupalmente a ambos), en la

(c) BY-NC-ND 2020, Universitat Politècnica de València 
comprensión y definición correcta conceptos relevantes relacionados con el contenido teórico-práctico de radiología (Fig. 4). Una de las ventajas del aprendizaje grupal y colaborativo es que cuando el alumnado comparte conocimientos desarrolla además ciertas habilidades y valores del comportamiento grupal, tales como serían el liderazgo, razonamiento crítico, comunicación asertiva y empática, etc. que son imprescindibles para los profesionales de Ciencias de la Salud (Castellanos-Nora, 2002; Valera-Ruiz, 2009).

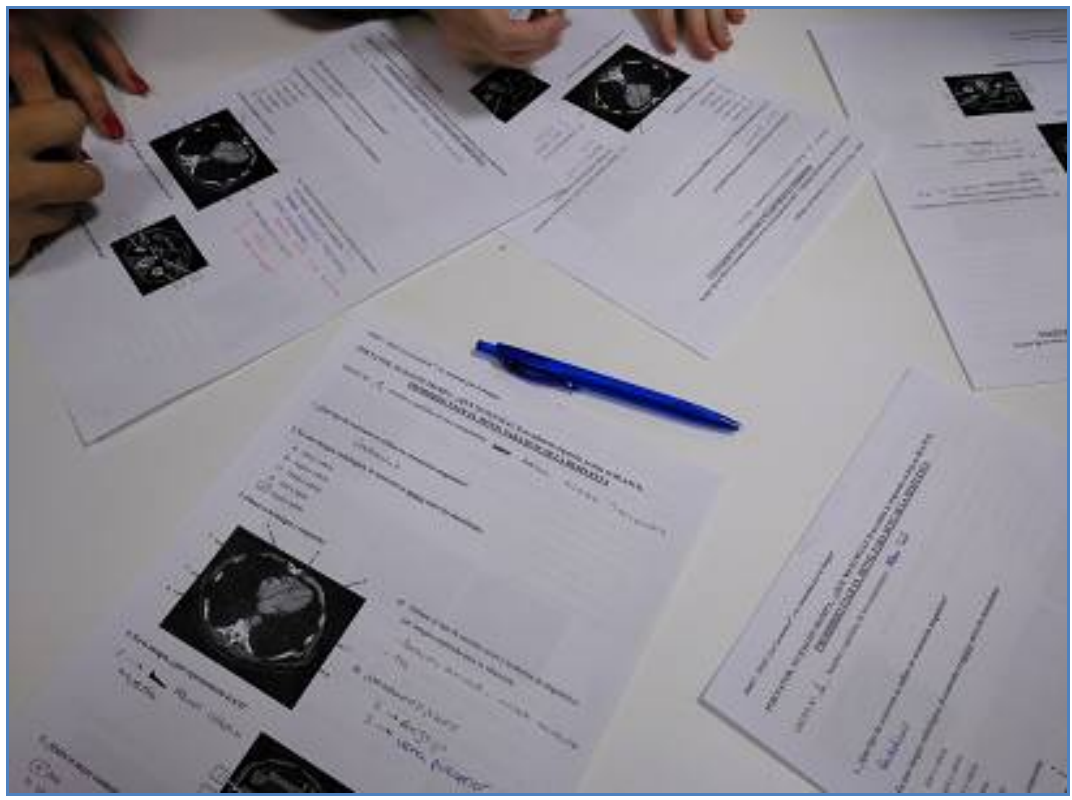

Fig. 4 Resolución de los cuestionarios post-juego de manera grupal.

Se han comparado las puntuaciones en ambos ensayos, antes y después del juego, utilizando estadísticos paramétricos y no paramétricos para la consecución del objetivo específico planteado inicialmente (identificar si existen diferencias significativas entre el aprendizaje individual y/o grupal tras la experiencia de ludificación). Se detectó la significación estadística con el programa SPSS Statistic 24. Además, dicho programa ha sido utilizado también para describir los resultados de un cuestionario tipo Likert en el que los estudiantes mostraron sus preferencias en una escala del 1 a 5 opinando sobre diferentes aspectos: actitud frente a la ludificación, valoración de las herramientas (analógicas y digitales), valoración de la aplicación de los contenidos teórico-prácticos en el juego, desarrollo de las competencias durante el juego y, por último, la valoración de la inmersión y diversión. El cuestionario de satisfacción también contaba con una pregunta abierta donde los participantes podían dejar su opinión sobre lo que más les había gustado o posibles sugerencias.

\section{Resultados}

Tras analizar los datos obtenidos de los cuestionarios teóricos antes y después del juego, se detectaron diferencias significativas en los conocimientos teórico-prácticos posteriores al juego, tanto en los alumnos del grado de TO como en los alumnos de FP (Fig.5):

○ En TO, como se trató desde un primero momento de un juego en equipo, se valoraron los conocimientos del alumnado en equipo: 
$\mu$ antes $=2,5 \pm 1,22$ y $\mu$ después $=4,025 \pm 1,77$. test de Wilcoxon, rechazo de $\mathrm{H}_{0}, \mathrm{p}=0,005<$ 0,005 . Se observó un rango de mejora significativo del 46,58\%.

○ En FP:

Comparativa antes y después del juego de manera individual (modificación introducida respecto al alumnado de TO):

$\mu$ antes $_{\text {(ind) }}=4,11 \pm 1,96$ y $\mu$ después $_{(\text {ind) }}=5,52 \pm 2,11$. t-student $=-4,802, p=0,000<$ 0,005. Se observó un rango de mejora significativo del $25,4 \%$.

Debido a que se trató de un juego en equipo, se consideró importante poder reflejar la aportación como grupo y no sólo la individual, para poder establecer si hay un enriquecimiento conjunto:

- $\mu$ después $_{(\text {ind) }}=5,52 \pm 2,11$ y $\mu$ después $_{(\text {grupal })}=7,83 \pm 1,71$. test de Wilcoxon, rechazo de $\mathrm{H}_{0}, \mathrm{p}=0,001<0,005$. Se observó un rango de mejora significativo del $29,6 \%$.

- $\mu \operatorname{antes}_{(\text {ind) }}=4,12 \pm 1,96$ y $\mu$ después $_{(\text {grupal }}=7,83 \pm 1,71$. test de Wilcoxon, rechazo de $\mathrm{H}_{0}, \mathrm{p}=0,001<0.005$. Se observó un rango de mejora significativo del $47,4 \%$.

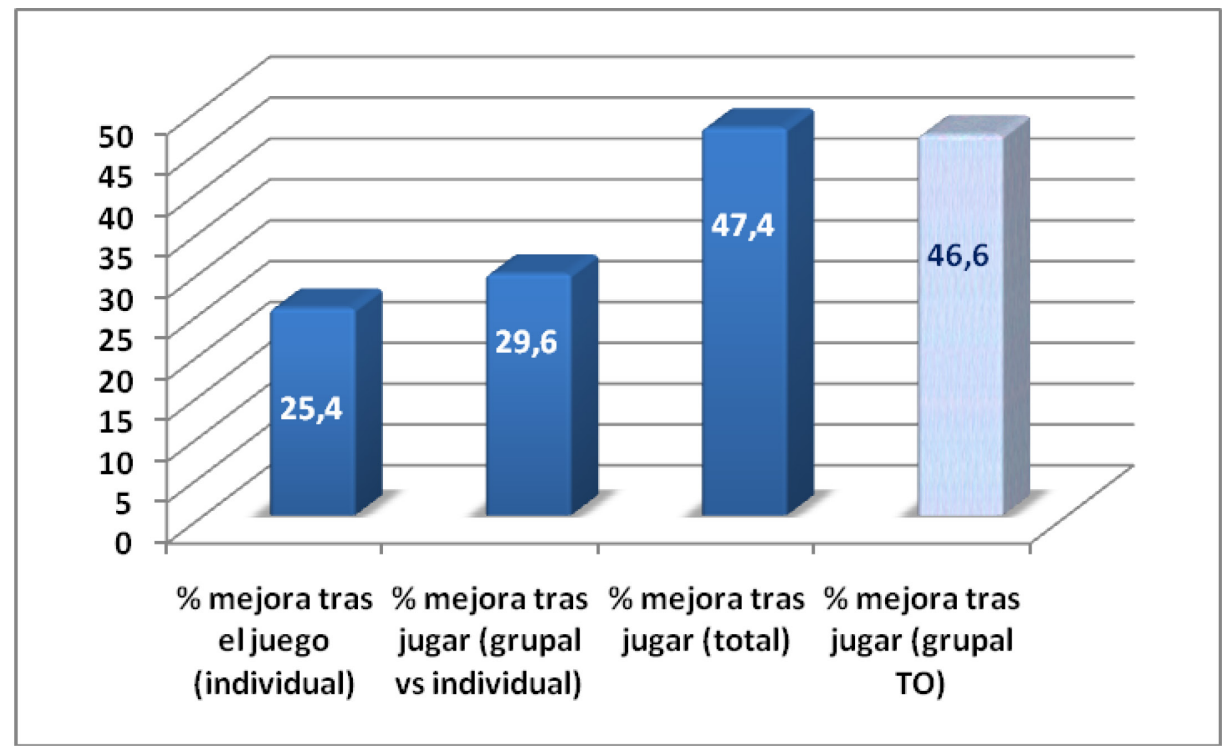

Fig. 5 La gráfica muestra el porcentaje del rango de mejora en los resultados obtenidos en los estudiantes de FP en los cuestionarios realizados, tanto de manera individual como grupal (barras de color azul oscuro) y el porcentaje de rango de mejora de los cuestionarios antes y después de jugar grupales en los estudiantes de TO (barra de color azul celeste).

Para la consecución del objetivo específico "conocer la opinión de los estudiantes sobre la ludificación, aplicación de los conocimientos y competencias, así como de la herramienta usada para el aprendizaje", se elaboró una encuesta de satisfacción a través de "Formularios de Google". Se utilizó una escala de tipo Likert: el alumnado respondió eligiendo entre una escala graduada y ordenada, siendo 1 muy en desacuerdo y 5 muy de acuerdo. Además, también se incluyó un apartado donde los participantes podían dejar su opinión acerca de lo que más les gustó y las posibles sugerencias, con el fin de obtener una mayor información de la metodología y del entorno de aprendizaje. El formulario fue de carácter 
voluntario, por lo que se obtuvo una participación del 73,59\%. Se obtuvieron un total de 39 respuestas del alumnado participante $(\mathrm{n}=53)$ : 16 respuestas de FP $(\mathrm{el} 100 \%)$ y 23 respuestas de TO $(62,16 \%)$.

Los resultados globales obtenidos de la encuesta del juego se exponen en la tabla 2 y muestran un alto grado de satisfacción, con puntuaciones superiores a 3,5 sobre 5 puntos en todos los aspectos relacionados con los parámetros analizados. Se muestra no solo la media sino también el valor de la moda con su frecuencia de elección.

Tabla 2: Resultados de la encuesta de satisfacción, que incluye media (M), desviación estándar (DE) y moda (Mo) con su frecuencia (N) y porcentaje (\%).

\begin{tabular}{lccr}
\hline $\begin{array}{l}\text { Ludificación: Indica tu grado de acuerdo con las siguientes } \\
\text { afirmaciones (1 Muy en desacuerdo - 5 Muy de acuerdo) }\end{array}$ & M (DE) & Mo & N (\%) \\
\hline 1) Me gusta jugar & $4,33(0,898)$ & 5 & $20(51,3)$ \\
2) Aprendo jugando & $4,15(0,961)$ & 4 & $22(56,4)$ \\
3) Los juegos me motivan a aprender & $4,31(0,863)$ & 4 & $18(46,2)$ \\
4) Prefiero los juegos en equipo & $4,13(1,056)$ & 5 & $19(48,7)$ \\
5) Los juegos educativos no son una pérdida de tiempo & $4,54(0,884)$ & 5 & $27(69,2)$
\end{tabular}

\begin{tabular}{llllll}
\hline Valoración de las herramientas, contenidos y materiales: & M $(D E)$ & Mo & N (\%)
\end{tabular}
Indica tu opinión general (1 Muy pobre- 5 Excelente)

\begin{tabular}{lccc}
\hline 6) App de realidad aumentada & $4,23(0,872)$ & 5 & $18(46,2)$ \\
7) Casos clínicos & $4,44(0,852)$ & 5 & $24(61,5)$ \\
8) Quiz o test progresivo & $4,38(0,815)$ & 5 & $11(53,8)$ \\
9) Teoría proporcionada & $3,82(1,114)$ & 5 & $14(35,9)$ \\
10) Evolución y desarrollo del juego & $4,46(0,854)$ & 5 & $25(64,1)$
\end{tabular}

Conocimientos y motivación: Indica tu grado de acuerdo con M $(D E)$ Mo N (\%) las siguientes afirmaciones (1 Muy en desacuerdo - 5 Muy de acuerdo)

\begin{tabular}{lccc}
\hline 11) El juego me ayudó a incrementar mis conocimientos & $4,03(0,903)$ & 4 & $20(51,3)$ \\
$\begin{array}{l}\text { 12) Fui capaz de integrar mis conocimientos de anatomía en el } \\
\text { juego }\end{array}$ & $4,00(0,761)$ & 4 & $23(59)$ \\
$\begin{array}{l}\text { 13) Jugar ha conseguido que quiera aprender más del diagnóstico } \\
\text { por imagen y anatomía radiológica }\end{array}$ & $3,79(0,894)$ & 4 & $18(46,2)$ \\
$\begin{array}{l}\text { 14) Gracias al juego puedo diferenciar las principales técnicas de } \\
\text { diagnóstico por imagen }\end{array}$ & $3,92(0,774)$ & 4 & $22(56,4)$ \\
$\begin{array}{l}\text { 15) Tras jugar, soy capaz de comprender qué técnica de } \\
\text { diagnóstico es más efectiva ante diferentes situaciones }\end{array}$ & $3,9(0,754)$ & 4 & $20(51,3)$ \\
16) Considero que el juego ha sido útil y he aprendido & $4,23(0,742)$ & 5 & $19(48,7)$
\end{tabular}




\begin{tabular}{lccc}
\hline $\begin{array}{l}\text { Competencias: ¿Qué competencias crees que has desarrollado } \\
\text { durante el juego? (1 Muy en desacuerdo - 5 Muy de acuerdo) }\end{array}$ & M (DE) & Mo & N (\%) \\
\hline 17) Habilidades interpersonales (de comunicación) & $4,13(0,812)$ & 4 & $19(48,7)$ \\
18) Trabajo en equipo & $4,15(0,812)$ & 4 & $21(53,8)$ \\
19) Capacidad de crítica y autocrítica & $4,15(0,709)$ & 4 & $22(56,4)$ \\
20) Resolución de problemas & $4,21(0,801)$ & 4 & $21(53,8)$ \\
21) Toma de decisiones & $4,15(0,844)$ & 4 & $19(48,7)$ \\
22) Capacidad para adaptarse a nuevas situaciones & $4,13(0,732)$ & 4 & $18(46,2)$ \\
23) Planificación y gestión del tiempo & $3,90(0,912)$ & 4 & $18(46,2)$ \\
24) Capacidad de análisis y síntesis & $4,03(0,811)$ & 4 & $20(51,3)$ \\
25) Aprendizaje autónomo & $4,10(0,680)$ & 4 & $24(61,5)$
\end{tabular}

\begin{tabular}{|c|c|c|c|}
\hline $\begin{array}{l}\text { Inmersión y diversión: Indica tu grado de acuerdo con las } \\
\text { siguientes afirmaciones ( } 1 \text { Muy en desacuerdo }-5 \text { Muy de } \\
\text { acuerdo) }\end{array}$ & $\bar{M}(\mathrm{DE})$ & Mo & $\mathbf{N}(\%)$ \\
\hline $\begin{array}{l}\text { 26) Mientras jugaba, tenía deseos de conseguir completar el } \\
\text { juego }\end{array}$ & $4,59(0,637)$ & $\overline{5}$ & $26(66,7)$ \\
\hline 27) Quería explorar todas las posibles opciones del juego & $4,15(0,812)$ & 4 & $16(41)$ \\
\hline 28) Sentí que el tiempo pasaba deprisa mientras jugaba & $4,46(0,682)$ & 5 & $22(56,4)$ \\
\hline 29) Estaba emocionada o emocionado mientras jugaba & $4,31(0,766)$ & 5 & $19(48,7)$ \\
\hline $\begin{array}{l}\text { 30) Me sentí parte de la historia del juego, quedando absorto en } \\
\text { él }\end{array}$ & $4,13(0,767)$ & 4 & $16(41)$ \\
\hline 31) Me divertí mientras jugaba & $4,41(0,595)$ & 4 & $19(48,7)$ \\
\hline
\end{tabular}

Para interpretar de manera descriptiva las medidas de tendencia central y su variabilidad en conjunto y no de manera aislada (Gómez, 2006), se analizaró por separado las secciones que componían el cuestionario (tabla 3). De esta forma, se determinó:

- Ludificación (compuesta por los items 1 al 5) y herramientas (compuesta por los itemas del 6 al 10): En ambas secciones, las puntuaciones tienden a agruparse en valores medios o elevados; por tanto, la actitud frente a la ludificación y la valoración de las herramientas resultó favorable de manera global y tanto en estudiantes de TO como de FP. La categoría que más se repitió en ambas secciones fue 5 (la máxima valoración). El valor mínimo registrado fue 1, en los estudiantes de TO; mientras que el valor máximo alcanzado fue 5 en todos los casos. En promedio, se obtiene una valoración por encima del 4 (actitud favorable). El valor del rango nos muestra que los datos presentan una gran dispersión, sobre todo, en el grupo de estudiantes de TO. Existen diferencias significativas tanto en la actitud frente a la ludificación entre estudiantes de TO y FP (prueba U-Mann-Whitney para muestras independientes, $p=0,013$ ) como en la 
valoración de las herramientas utilizadas, (prueba U-Mann-Whitney para muestras independientes, $\mathrm{p}=0,01)$.

- Conocimientos (compuesta por los items 11 al 16): Las puntuaciones tienden a agruparse en valores medios o poco elevados; por tanto, la valoración de los conocimientos adquiridos resultó favorable de manera global y tanto en estudiantes de TO como de FP. La categoría que más se repitió fue 4 (de acuerdo o favorable). El valor mínimo registrado fue 1, en los estudiantes de TO; mientras que el valor máximo alcanzado fue 5 en todos los casos. En promedio, se obtiene una valoración próxima a 4 (actitud favorable). De nuevo, el valor del rango nos muestra que los datos presentan una gran dispersión, sobre todo, en el grupo de estudiantes de TO. Además, la prueba U de Mann-Whitney para muestras independientes determinó que existían diferencias significativas en la valoración de la aplicación de los conocimientos entre los estudiantes de TO y FP $(\mathrm{p}=0,02)$.

- Competencias (compuesta por los items 17 al 25): Las puntuaciones tienden a agruparse en valores medios o elevados; por tanto, la valoración de las competencias trabajadas resultó favorable de manera global y tanto en estudiantes de TO como de FP. La categoría que más se repitió en ambas secciones fue 4 (de acuerdo o favorable). El valor mínimo registrado fue 1, en los estudiantes de TO; mientras que el valor máximo alcanzado fue 5 en todos los casos. En promedio, se obtiene una valoración por encima del 4 (actitud favorable). El valor del rango nos muestra de nuevo que los datos presentan una gran dispersión, sobre todo, en el grupo de estudiantes de TO. No obstante, no se han encontrado diferencias estadísticamente significativas en la valoración de las competencias trabajadas entre estudiantes de grado y de formación profesional (U Mann-Whiyney para muestras independiente, $\mathrm{p}=0,159$ ).

- Inmersión y diversión (compuesta por los items 26 al 31): Las puntuaciones tienden a agruparse en valores medios o elevados; por tanto, la valoración de las competencias trabajadas resultó favorable de manera global y tanto en estudiantes de TO como de FP. Sin embargo, hubo diferencias de la valoración de la inmersión y diversión estadísticamente significativas (prueba $\mathrm{U}$ Mann-Whitney, $\mathrm{p}=0$,035) La categoría que más se repitió en ambas secciones fue 4 (de acuerdo o favorable). El valor mínimo registrado fue 2, en los estudiantes de TO; mientras que el valor máximo alcanzado fue 5 en todos los casos. En promedio, se obtiene una valoración por encima del 4 (actitud favorable). En esta sección la dispersión de los datos es ligeramente menor que en las restantes, aunque sigue observándose que es en los estudiantes de grado donde es más acusada.

Tabla 3: Resultados de la encuesta de satisfacción agrupados por variables, que determinan la actitud global y de los estudiantes de FP y de TO por separado. Incluyen moda y el porcetaje de dicho valor dentro de cada categoría (Mo(\%)), la mediana (Me) la media con la desviación estándar (M(DE), el valor máximo (Máx.), el valor mínimo (Mín.) y el rango.

\section{Variable: Actitud frente a la ludificación (Îtems 1-5)}

\begin{tabular}{|l|ccc|}
\hline & GLOBAL & Estudiantes FP & Estudiantes TO \\
\hline Mo (\%) & $5(50,27)$ & $5(66,25)$ & $4(44,35)$ \\
\hline Me & 5 & 5 & 4 \\
\hline M (DE) & $4,29(0,94)$ & $4,61(0,58)$ & $4,07(1,07)$ \\
Máx. & 5 & 5 & 5 \\
\hline
\end{tabular}




\begin{tabular}{|c|c|c|c|}
\hline Mín. & 1 & 3 & 1 \\
\hline Rango & 4 & 2 & 4 \\
\hline \multicolumn{4}{|c|}{ Variable: Valoración de las herramientas (Ítems 6-10) } \\
\hline & GLOBAL & Estudiantes FP & Estudiantes TO \\
\hline Mo (\%) & $5(52,31)$ & $5(63,75)$ & $5(44,35)$ \\
\hline Me & 5 & 5 & 4 \\
\hline M (DE) & $4,27(0,94)$ & $4,61(0,54)$ & $4,03(1,07)$ \\
\hline Máx. & 5 & 5 & 5 \\
\hline Mín. & 1 & 3 & 1 \\
\hline Rango & 4 & 2 & 4 \\
\hline \multicolumn{4}{|c|}{ Variable: Valoración de los conocimientos y motivación (Ítems 11-16) } \\
\hline & GLOBAL & Estudiantes FP & Estudiantes TO \\
\hline Mo (\%) & $4(62,56)$ & $4(58,75)$ & $4(59,13)$ \\
\hline Me & 5 & 4 & 4 \\
\hline M (DE) & $3,98(0,81)$ & $4,33(0,57)$ & $3,73(0,86)$ \\
\hline Máx. & 5 & 5 & 5 \\
\hline Mín. & 1 & 3 & 1 \\
\hline Rango & 4 & 2 & 4 \\
\hline \multicolumn{4}{|c|}{ Variable: Valoración de las competencias aplicadas (Ítems 17-25) } \\
\hline & GLOBAL & Estudiantes FP & Estudiantes TO \\
\hline Mo (\%) & $4(93,33)$ & $4(45)$ & $4(77,39)$ \\
\hline Me & 4 & 4 & 4 \\
\hline M (DE) & $4,11(0,78)$ & $4,26(0,54)$ & $4(0,90)$ \\
\hline Máx. & 5 & 5 & 5 \\
\hline Mín. & 1 & 3 & 1 \\
\hline Rango & 4 & 2 & 4 \\
\hline \multicolumn{4}{|c|}{ Variable: Valoración la inmersión y diversión (Ítems 26-31) } \\
\hline & GLOBAL & Estudiantes FP & Estudiantes TO \\
\hline Mo (\%) & $5(58,46)$ & $5(75)$ & $4(49,57)$ \\
\hline Me & 4 & 5 & 4 \\
\hline M (DE) & $4,34(0,73)$ & $4,56(0,61)$ & $4,19(0,76)$ \\
\hline Máx. & 5 & 5 & 5 \\
\hline Mín. & 2 & 3 & 2 \\
\hline Rango & 3 & 2 & 3 \\
\hline
\end{tabular}


Lo comentarios recibidos en la pregunta abierta del cuestionario de satisfacción, así como la implicación de los estudiantes y profesores durante el desarrollo del juego, determinan que se alcanzaron los objetivos específicos del estudio. A continuación se muestran algunos de los comentarios recibidos como respuesta la pregunta abierta:

De Terapeutas Ocupacionales:

“...me ha gustado mucho el diseño del juego”.

"La imnersión del juego en equipo y usar la realidad aumentada para saber las respuestas, fue lo mejor".

"Me gustó mucho el juego Lucusines, ya que podíamos tomar decisiones como si fuera un caso clínico real".

"Lo mejor ha sido poder jugar y avanzar trabajando en equipo, aunque al principio pensé que nuestro grupo iba a tener problemas a la hora de tomar decisiones pero al final nos pusimos todos de acuerdo".

"A principio no entendía nada, pero luego he aprendido sobre un tema del que no tenia conocimiento alguno".

"Me ha encantado la dinámica del juego y el uso de la aplicación de realidad aumentada".

"Me ha sorprendido porque pensaba que el juego iba a ser mucho mas aburrido y me he divertido".

"Lo que menos me ha gustado ha sido la competencia entre equipos y lo que más, los casos clínicos".

“... me ha gustado aprender de una manera diferente sobre una temática que desconocía”.

De estudiantes de Formación Profesional:

"No me esperaba que fuera un juego tan educativo y elaborado; me gustó que el caso clínico se viera a través del móvil".

"Me ha ayudado a aprender a trabajar en equipo y a fijar conocimientos".

“Me gustó mucho esta práctica para aprender y fijar conocimientos; me divertí aprendiendo".

"Creo que aprendemos mejor si estamos entretenidos y participando activamente, por eso me ha gustado".

"Lo que más me ha gustado ha sido trabajar en equipo, pero la aplicación no me gustó porque fallaba en mi móvil”.

"No me ha gustado que los grupos estuvieran tan próximos porque escuchaban nuestras respuestas".

"Lo que menos me agradó fueron las discusiones que se produjeron dentro del grupo, aunque al final se consiguió trabajar en equipo".

"A veces la aplicación fallaba, por lo que nos retrasaba en el avance del juego".

\section{Conclusiones}

Si bien se tratan de dos estudios piloto a pequeña escala, los hallazgos encontrados indican que iniciativas similares podrían ser relevantes para otras materias y estudios de Educación Superior, tanto universitaria como no universitaria. 
Tras concluir el juego propuesto, los estudiantes encontraron que fue una experiencia de aprendizaje efectiva e innovadora para la aplicación de conocimientos y desarrollo de competencias, como la capacidad del trabajo en equipo, que ayudarán significativamente al éxito en su desempeño profesional. Se observa un incremento de mejora en las puntuaciones de los test teórico-prácticos que es aún más acusada, tras la resolución como grupo, después de haber colaborado para llegar a la resolución de los problemas planteados.

En lo que respecta a la actitud frente a la ludificación como método de aprendizaje, la valoración de los conocimientos empleados durante el juego y la valoración de la inmersión y diversión del juego, los alumnos de grado y de formación profesional mostraron diferencias estadísticamente significativas, presentando unos valores ligeramente más elevados los estudiantes de formación profesional. Esto puede ser por varios motivos. Uno de los que se baraja es que cuando se jugó por segunda vez, ya se había arreglado ciertos inconvenientes que se observaron en el primer juego. Otro de los motivos, tal vez fuera, que los contenidos del juego eran más afines y conocidos por el alumnado de formación profesional del ciclo superior de Imagen para el Diagnóstico y Medicina Nuclear, por lo que enseguida se hicieron con la dinámica del juego. Esto también puede verse reflejado en los resultados obtenidos en los cuestionarios teórico-prácticos tanto antes como después del juego, en el que se observa que los estudiantes de formación profesional obtuvieron mejores resultados.

Sin embargo, en la valoración de las competencias empleadas, no se encontraron diferencias estadísticamente significativas entre ambos perfiles de alumnos.

Los datos obtenidos a partir de las encuestas de satisfacción se tendrán presentes para mejorar el diseño y contenido del juego. Por ejemplo, se buscarán alternativas al uso de la App, porque es cierto que a veces daba error y se requería de conexión wifi para poder jugar, entre otras cosas.

Por otra parte, también se ha de resaltar que el desarrollo e implementación del juego exigió capacidad de organización y colaboración entre los profesores, alumnado y centros (Facultad Padre Ossó en el caso del Grado en Terapia Ocupacional y Svida Fornación, en el caso de los alumnos de Formación Profesional del Ciclo Superior de Imagen para el Diagnóstico y Medicina Nuclear), para la elección del día del juego y la toma de decisiones sobre cuál era el mejor momento para implementarlo y con qué contenidos.

\section{Agradecimientos}

Agradecer la participación del alumnado de $1^{\circ}$ de Terapia Ocupacional de la Facultad Padre Ossó de la y del alumnado de $1^{\circ}$ de Imagen para el Diagnóstico y Medicina Nuclear del Centro de Formación Profesional Svida Formación.

\section{Referencias}

ANDREU-ANDRÉS Ma. \& GARCÍA-CASAS M. (2014). “Gaming in higher education: student's assessment on game-based learning”. En 45th Conference of the International Simulation and Gaming Association (ISAGA). Dronbirn, Austria. W. Bertelsmann Verlag Gmbh \& Co. Bielefeld, Germany. CD-Rom, p. 33-44. ISBN: 978-3- 7639-54213.

BAID, H., LAmBERT, N. (2010). "Enjoyable learning: the role of humour, games, and fun activities in nursing and midwifery education”. Nurse Education Today, 30, 548-552. https://dx.doi.org/10.1016/j.nedt.2009.11.007.

Castellanos-Noda V. (2002). "La actividad de aprendizaje grupal”. Revista Cubana de Psicología, 19 (2), $99-105$. 
CONTRERAs-EsPinOZA R. (2016). “Juegos digitales y gamificación aplicados en el ámbito de la educación”. En RIED: Revista Iberoamericana de Educación a Distancia, 19 (2), 27-33. http://dx.doi.org/10.5944/ried.19.2.16143.

Domínguez, A., SÁenz-de-Navarrete, J., deMarcos, L., Fernández-Sanz, L., Pagés, C. \& Martínez-Herráiz, J.J. (2013). "Gamifying learning experiences: Practical implications and outcomes". Computers and Education, 63(1), 380-392. https://dx.doi.org/10.1016/j.compedu.2012.12.020.

ERRINGTON E. (2001). The influence of teacher beliefs on flexible learning innovation in traditional university settings. Innovation in open and distance learning. Successful development of online and web-based learning, Kogan Page, London, UK, pp. 27-37.

Filippou J, Cheong C, Cheong F. (2018). "A model to investigate preference for use of gamification in a learning activity”. Australasian Journal of Information Systems, 22, 1-23. https://doi.org/10.3127/ajis.v22i0.1397.

FUKUCHI ET AL. (2000) “Teaching a Multidisciplinary Approach to Cancer Treatment during Surgical Clerkship via an Interactive Board Game”. The American Journal of Surgery. 179, 337-340. https://doi.org/10.1016/s00029610(00)00339-1.

Gómez M.M. (2006). Introducción a la metodología de la investigación científica, Brujas, Córdoba, pp. 154-155.

Hoffman B., \& NADELSON L. (2009). "Motivational engagement and video gaming: a mixed methods study". Educational Technology Research and Development, 58, 245-270. https://doi.org/10.1007/s11423-009-9134-9.

KAPP K.M. (2012). The gamification of learning and instruction: game based methods and strategies for training and education. New York: Pfeiffer-Wiley.

RUE J. (2009). El aprendizaje autónomo en la Educación Superior. Madrid: Narcea.

Rutledge C.; Walsh C.M.; Swinger N., Auerbach, M.; Castro D., Dewan, M.; Khattab, M.; Rake, A.; HARWAyne-GidAnSky I.; RAYMOND T.T.; MAA T. \& CHANG, T.P. (2018). "Gamification in action: theoretical and practical considerations for medical educators”. Academic Medicine, 93(7), 1014-1020. https://doi.org/10.1097/ACM.0000000000002183.

VALERA-RUIZ M. (2009). “Aprendizaje independiente y aprendizaje colaborativo en educación médica" Revista Médica del Hospital General de México, 72(4), 222-227.

Villalustre Martínez L.; Del Morán L.E. (2015). “Gamificación: Estrategia para optimizar el proceso de aprendizaje y la adquisición de competencias en contextos universitarios" en Digital Education Review. Barcelona: Revistes Cientifiques de la Universitat de Barcelona, 27, 13-31. http://greav.ub.edu/der [Consulta: 10 de marzo de 2020]. 